\title{
COMUNICAÇÃO INTERPESSOALDO ENFERMEIRO DURANTE O EXAME FÍSICO: FATORES QUE INTERFEREM NESTA COMPETÊNCIA
}

Ana Elise Lopes Pontes ${ }^{1}$ Francine da Costa Alves ${ }^{2}$ Thais Josgrilberg Pereira ${ }^{3}$ Ana Claudia Giesbrecht Puggina https://orcid.org/0000-0001-6523-2994 https://orcid.org/0000-0002-3768-9476 https://orcid.org/0000-0001-7806-5398

https://orcid.org/0000-0001-8095-6560

Objetivo: avaliar quais fatores sociodemográficos e profissionais interferem na competência em comunicação interpessoal do enfermeiro durante o exame físico. Metodologia: Estudo analítico, transversal, quantitativo realizado com enfermeiros assistenciais. Foi utilizada a “Escala de Autoavaliação sobre Profissionalismo e Comunicação Interpessoal entre Enfermeiros e Pacientes". Resultados: A amostra constituiu-se de 171 enfermeiros com média de idade 33,93 anos ( $\pm 7,14 \%)$, a maioria do sexo feminino (87,7\%) e com companheiro estável (63,1\%). 0 escore médio total foi de 47,7 ( $\pm 5,1)$. Houve diferença estatística na comparação entre a cor autorreferida com o fator sinceridade nas relações ( $p=0,04)$ e na comparação entre a idade e o fator habilidade interpessoal ( $p=0,05)$. Conclusões: Os enfermeiros percebem-se com moderado a alto profissionalismo e competentes na relação enfermeiro-paciente. Os fatores que interferiram na comunicação interpessoal e profissionalismo do enfermeiro foram: idade, cor autorreferida, tempo de formação, escolaridade, religião, sexo e unidade de atuação.

Descritores: Enfermagem; Exame Físico; Comunicação; Relações Enfermeiro-Paciente.

\section{INTERPERSONAL COMMUNICATION OF THE NURSE DURING THE PHYSICAL EXAMINATION: FACTORS THAT INTERFERE IN THIS COMPETENCE}

Objective: to evaluate which sociodemographic and professional factors interfere in nurses' competence in interpersonal communication during physical examination. Methodology: Quantitative, cross-sectional, analytical study performed with nursing assistants. The "Self-Assessment Scale on Professionalism and Interpersonal Communication between Nurses and Patients" was used. Results: The sample consisted of 171 nurses with a mean age of 33.93 ( $\pm 7.14 \%$ ), the majority of whom were female (87.7\%) and had a stable partner (63.1\%). The mean total score was 47.7 ( \pm 5.1$)$. There was a statistical difference in the comparison between the self-reported color and the sincerity factor in the relationships ( $p=0.04)$ and in the comparison between age and the interpersonal ability factor $(p=0.05)$. Conclusions: Nurses perceive themselves with moderate to high professionalism and competent in the nurse-patient relationship. The factors that interfered in the interpersonal communication and professionalism of the nurse were: age, self-referenced color, training time, schooling, religion, sex and unit of performance.

Descriptors: Nursing; Physical Examination; Communication; Nurse-Patient Relations.

\section{COMUNICACIÓN INTERPERSONAL DEL ENFERMERO DURANTE EL EXAMEN FÍSICO: FACTORES QUE INTERFEREN EN ESTA COMPETENCIA}

Objetivo: evaluar qué factores sociodemográficos y profesionales interfieren en la competencia en comunicación interpersonal del enfermero durante el examen físico. Metodologia: Estudio analítico, transversal, cuantitativo realizado con enfermeros asistenciales. Se utilizó la “Escala de Autoevaluación sobre Profesionalismo y Comunicación Interpersonal entre Enfermeros y Pacientes". Resultados: La muestra se constituyó de 171 enfermeros con promedio de edad 33,93 años ( \pm 7,14\%), la mayoría del sexo femenino (87,7\%) y con compañero estable (63,1\%). La puntuación media total fue de 47,7 ( $\pm 5,1)$. Se observó una diferencia estadistica en la comparación entre el color autorreferido con el factor sinceridad en las relaciones ( $p$ = 0,04) y en la comparación entre la edad y el factor habilidad interpersonal $(p=0,05)$. Conclusiones: Los enfermeros se perciben con moderado a alto profesionalismo y competentes en la relación enfermero-paciente. Los factores que interfirieron en la comunicación interpersonal y profesionalismo del enfermero fueron: edad, color autorreferido, tiempo de formación, escolaridad, religión, sexo y unidad de actuación

Descriptores: Enfermería; Examen Físico; Comunicación; Relaciones Enfermero-Paciente.

${ }^{1}$ Instituto do Coração do Hospital das Clínicas da Faculdade de Medicina da Universidade de São Paulo - HCFMUSP, SP ${ }^{2}$ Universidade de Taubaté - UNITAU, SP

${ }^{3}$ Faculdade de Educação e Cultura de Vilhena - FAEV, RO

${ }^{4}$ Faculdade de Medicina de Jundiai - FMJ, SP

Autor correspondente: Ana Claudia Giesbrecht Puggina - E-mail: claudiagpuggina@gmail.com 


\section{INTRODUÇÃO}

Comunicação é o processo de compreender e compartilhar ideias, uma importante necessidade humana, por meio da qual os indivíduos partilham sentimentos, crenças, valores, atitudes e comportamentos. É a forma de interação humana por meio da qual iniciam-se as relações interpessoais, entre duas pessoas, e há a tentativa de entender o outro e de se fazer compreendido ${ }^{(1-2)}$.

Para comunicar-se o indivíduo usa basicamente dois tipos de comunicação, a verbal e a não verbal. A linguagem verbal relaciona-se diretamente as palavras, podendo ser escrita ou falada, e a não verbal caracteriza-se por informações/mensagens emitidas ou recebidas por gestos, posturas, expressões faciais, orientações do corpo, tom e ritmo da voz, organização dos objetos no espaço e até mesmo pela relação de proximidade e distância entre as pessoas $^{(1)}$.

A comunicação é um instrumento básico do cuidar e nas atividades do enfermeiro ela é um elemento por meio da qual se estabelece a relação profissional-paciente; ambos se conhecem, descobrem-se, se percebem, ensinam e aprendem um com outro, cada um com o seu contexto e suas experiências, sendo assim, com essa troca, é possivel a prática do cuidar ${ }^{(3)}$.

As etapas do cuidar são fenômenos comunicativos entre enfermeiro e paciente sendo essencial que o profissional possua habilidades de comunicação necessárias tanto para transmitir informações claras e compreensiveis para o paciente, quanto para ouvir, escutar, observar e interpretar o outro na sua complexidade ${ }^{(3)}$.

O cuidar requer ação interativa e envolvimento. Essa ação está alicerçada em valores morais e no conhecimento do ser que cuida "para" e "com" o ser que é cuidado. O cuidado é capaz de ativar comportamentos de compaixão, de solidariedade, de ajuda, no sentido de promover o bem-estar do paciente, manter sua integridade moral e dignidade como pessoa ${ }^{(4)}$.

A anamnese e o exame físico talvez representem os momentos de maior interação enfermeiro-paciente. É o momento em que o enfermeiro inicia o processo de um cuidado individualizado, holístico, humanizado, com embasamento científico e centrado no paciente, portanto negligenciar essa etapa do cuidado, tanto do ponto de vista comunicacional quanto técnico-científico, pode impactar negativamente na assistência de enfermagem.

A contextualização e a identificação correta dos problemas apresentados pelos pacientes são fundamentais para o desenvolvimento das ações do enfermeiro para prevenir, promover, proteger, recuperar e manter a saúde dos pacientes ${ }^{(5)}$. Sem dúvida, a comunicação fundamenta todo esse processo e pode promover uma atuação mais adequada, criteriosa e efetiva do cuidar

Considerando a importância da comunicação enfermeiro-paciente para o cuidado de enfermagem, o objetivo deste estudo foi avaliar quais fatores sociodemográficos e profissionais interferem na competência em comunicação interpessoal do enfermeiro durante o exame físico.

\section{METODOLOGIA}

\section{Tipo de estudo}

Estudo analítico, transversal, sob abordagem quantitativa.

\section{Participantes da pesquisa}

Foram incluidos no estudo enfermeiros assistenciais convidados a participar da pesquisa por meio de recrutamento indireto no ambiente virtual por meio da rede social Facebook ${ }^{\circledR}$ e que atuavam nas áreas hospitalar, ambulatorial ou da atenção básica.

Para confirmar que o participante era enfermeiro fo solicitado a identificação com o nome completo e número de inscrição no Conselho Regional de Enfermagem (COREN), e posteriormente foi conferido nos sites dos conselhos regionais esta informação. Os participantes que não eram enfermeiros e que não atendiam aos critérios de inclusão, bem como profissionais que responderam mais de uma vez foram excluídos da amostra.

Foram excluídos enfermeiros que estavam atuando em cargos administrativos, tais como coordenadores e gerentes, por, em geral, não realizarem ou realizar menos frequentemente exame físico nos pacientes e também foram excluidos enfermeiros assistenciais com atuação em unidades pediátricas e neonatais, pois a assistência e a comunicação com a criança e sua família difere muito do adulto, contexto proposto pelo instrumento utilizado neste estudo. Essas exclusões foram feitas mediante respostas dos participantes em relação a atuação no momento da pesquisa.

O cálculo amostral foi determinado pela média e desvio padrão obtidos no estudo de validação da "Escala de Autoavaliação sobre Profissionalismo e Comunicação Interpessoal entre Enfermeiros e Pacientes"(6). Considerando o escore médio $(47,8)$, o desvio padrão $( \pm 3,99)$, o erro máximo da estimativa correspondente a $15 \%$ do desvio padrão $(0,598)$ e fixando o nível de significância em $5 \%$, o tamanho amostral estimado para aplicação deste instrumento foi de 171 participantes. 


\section{Local do estudo}

O estudo foi realizado exclusivamente em ambiente virtual, na rede social Facebook ${ }^{\circledR}$. Um link foi publicado na linha do tempo dos grupos "Enfermagem", "Grupo de Estudo Enfermagem", “Enfermagem nota 10", “Enfermagem Atualizada e Reciclada", no perfil dos pesquisadores ou foi enviado por mensagem para enfermeiros que faziam parte da rede social com perfis pessoais vinculados aos pesquisadores.

\section{Coleta de dados}

A coleta de dados ocorreu entre maio e julho de 2016. Foram utilizados dois instrumentos para coleta de dados: um instrumento de caracterização e a "Escala de Autoavaliação sobre Profissionalismo e Comunicação Interpessoal entre Enfermeiros e Pacientes". Para isso, um questionário online foi construído com a ferramenta digital do Google Drive $^{\circledR}$. Esta ferramenta possibilitou que as respostas ficassem automaticamente disponiveis aos pesquisadores em uma planilha Excel ${ }^{\circledR}$

O instrumento de caracterização dos participantes continha as seguintes variáveis: idade, sexo, naturalidade, estado matrimonial, cor autorreferida, escolaridade, unidade de atuação, tempo de atuação e renda individual.

A "Escala de Autoavaliação sobre Profissionalismo e Comunicação Interpessoal entre Enfermeiros e Pacientes" é um instrumento que mede a comunicação interpessoal do enfermeiro durante o exame físico e configura-se em uma autoavaliação da maneira que, em geral, o profissional se comporta nessa situação(b).

Esta escala é composta por 11 itens distribuídos em 4 fatores, mensurados por meio de uma escala tipo Likert de cinco pontos que varia de "nunca" a "sempre". O escore total varia de 11 a 55 e quanto maior o escore, maior a autoavaliação e competência em comunicação interpessoal. O item 11, mensura o nível de profissionalismo com uma escala tipo Likert que difere dos demais itens: 1 baixo; 2 baixo a moderado; 3 moderado; 4 moderado a baixo e 5 alto(6).

O primeiro fator "Habilidade Interpessoal" é definido como a capacidade do indivíduo em reagir da melhor forma possivel a presença do outro incluindo gestos utilizados, tratamento a outra pessoa, escuta e interesse pelo outro, e constitui-se por 4 itens $(2,3,4,5)$. O fator "Troca de informações" define a capacidade do profissional em transmitir e receber informações pertinentes durante o exame físico, e é composto por 3 itens $(6,7,8)$. O fator "Sinceridade na relação" envolve a relação profissional entre enfermeiro e paciente, necessidade de falar ao paciente o que for pertinente, saber em cada momento durante o acompanha- mento de saúde e possui 2 itens (1, 9). O fator "Profissionalismo" define a capacidade do profissional em classificar o seu profissionalismo e isso é avaliado em 2 itens (10 e ll) $)^{(6)}$.

\section{Procedimentos de análise dos dados}

Foram realizadas análises descritiva (média, desvio-padrão e mediana) e comparativa, por meio de testes estatísticos pelo software IBM SPSS ${ }^{\circledR}$ versão 16. A análise comparativa bivariada foi feita por meio de testes estatísticos não paramétricos (teste de Mann-Whitney para dois grupos e teste de Kruskal-Wallis para três ou mais grupos). O nivel de significância adotado foi $\leq 5 \%$ e uma tendência de significância $\leq 12 \%$ foi também assumida pelos pesquisadores nas análises comparativas. Foi realizado o teste de qui-quadrado de Pearson para avaliar a correlação entre os fatores da escala e as variáveis numéricas.

\section{Procedimentos éticos}

Ao clicar no link, a primeira tela continha o Termo de Consentimento Livre e Esclarecido (TCLE), o consentimento do participante do estudo foi obtido por meio de aceite eletrônico, ou seja, o participante tinha que clicar em "aceito participar da pesquisa" para prosseguir, os que clicavam em "não aceito participar da pesquisa" eram direcionados a uma página com agradecimentos dos pesquisadores e o acesso era encerrado.

O desenvolvimento do estudo atendeu as normas nacionais e internacionais de ética em pesquisa envolvendo seres humanos e foi aprovado pelo Comitê de Ética e Pesquisa sob o Parecer $N^{\circ}$ 1.532.127.

\section{RESULTADOS}

A amostra do estudo foi de 171 enfermeiros, com média de idade igual a 33,93 anos $( \pm 7,14)$ e renda média de 4,26 $( \pm 1,58)$ salários mínimos. A maioria era do sexo feminino, com companheiro estável, católicos e brancos. Em relação as características profissionais, o tempo médio de formação igual a 6,78 $( \pm 5,4)$ anos. A maioria eram provenientes da Região Sudeste e maiores frequências foram observadas de profissionais com especialização e atuando em unidades de internação (Tabela 1).

Tabela 1. Descrição das características dos participantes da pesquisa. Jundiaí, SP, 2016.

\begin{tabular}{llllll} 
Caracteristicas & $\mathbf{n}$ & $\%$ & Caracteristicas & $\mathbf{n}$ & $\%$ \\
Sexo & & \multicolumn{5}{c}{$\begin{array}{c}\text { Naturalidade } \\
\text { Feminino }\end{array}$} & 150 & 87,7 & Sudeste & 137 & 80,1 \\
Masculino & 21 & 12,3 & Centro oeste & 13 & 7,6
\end{tabular}




\begin{tabular}{|c|c|c|c|c|c|}
\hline $\begin{array}{l}\text { Estado Matrimo- } \\
\text { nial }\end{array}$ & & & Sul & 9 & 5,3 \\
\hline $\begin{array}{l}\text { Com compa- } \\
\text { nheiro estável }\end{array}$ & 108 & 63,2 & Norte & 6 & 3,5 \\
\hline $\begin{array}{l}\text { Sem compa- } \\
\text { nheiro estável }\end{array}$ & 63 & 36,8 & Nordeste & 5 & 2,9 \\
\hline Religião & & & Outra & 1 & 0,6 \\
\hline Católico & 99 & 57,9 & Unidade de atuação & & \\
\hline Evangélico & 39 & 22,2 & $\begin{array}{l}\text { Unidade de In- } \\
\text { ternação }\end{array}$ & 69 & 40,4 \\
\hline Espirita & 19 & 11,1 & Pronto Socorro & 43 & 25,1 \\
\hline Outras* & 14 & 8,0 & $\begin{array}{l}\text { UTI ou Semi } \\
\text { Intensiva }\end{array}$ & 35 & 20,5 \\
\hline $\begin{array}{l}\text { Cor Autorrefe- } \\
\text { rida** }^{* *}\end{array}$ & & & Ambulatório & 14 & 8,2 \\
\hline Branca & 134 & 78,4 & $\begin{array}{l}\text { Unidade Básica } \\
\text { de Saúde }\end{array}$ & 10 & 5,8 \\
\hline Parda & 31 & 18,1 & Escolaridade & & \\
\hline Preta & 5 & 2,9 & Ensino Superior & 80 & 46,8 \\
\hline \multirow[t]{2}{*}{ Sem declaração } & 1 & 0,6 & Especialização & 81 & 47,4 \\
\hline & & & Mestrado & 10 & 5,8 \\
\hline Total & 171 & 100,0 & Total & 171 & 100,0 \\
\hline
\end{tabular}

*Outras religiões: Adventista $(n=2)$, Umbandista $(n=1)$, Testemunha de Jeová $(n=1)$, Mórmon $(n=1)$, Sem religião $(n=7)$, Outras $(n=2) .{ }^{*}$ Cor auto autorreferida: os grupos raciais foram classificados segundo IBGE Censo 2010. UTI: Unidade de Terapia Intensa.

O escore médio total das respostas dos participantes em relação a "Escala de Autoavaliação sobre Profissionalismo e Comunicação Interpessoal entre Enfermeiros e Pacientes" foi de $47,7( \pm 5,1)$; esse dado mostra que os profissionais apresentam uma percepção geral moderada a alta sobre seu profissionalismo e comunicação interpessoal (Tabela 2).

Tabela 2. Descrição do escore total e por fator da "Escala de Autoavaliação sobre Profissionalismo e Comunicação Interpessoal entre Enfermeiros e Pacientes". Jundiaí, SP,

\section{6.}

\begin{tabular}{|c|c|c|c|c|c|}
\hline & $\begin{array}{l}N^{\circ} \text { de } \\
\text { itens }\end{array}$ & $\begin{array}{l}\text { Varia- } \\
\text { ção do } \\
\text { escore }\end{array}$ & $\begin{array}{l}\text { Mé- } \\
\text { dia }\end{array}$ & $\begin{array}{l}\text { Des- } \\
\text { viopa- } \\
\text { drão }\end{array}$ & $\begin{array}{l}\text { Me- } \\
\text { diana }\end{array}$ \\
\hline $\begin{array}{l}\text { Habilidade Interpes- } \\
\text { soal }\end{array}$ & 4 & $4-20$ & 18,0 & 2,2 & 19,0 \\
\hline Troca de informações & 3 & $3-15$ & 12,2 & 2,2 & 13,0 \\
\hline $\begin{array}{l}\text { Sinceridade na re- } \\
\text { lação }\end{array}$ & 2 & $2-10$ & 8,2 & 1,2 & 8,00 \\
\hline Profissionalismo & 2 & $2-10$ & 8,7 & 0,8 & 9,00 \\
\hline TOTAL & 11 & $11-55$ & 47,0 & 5,1 & 39,0 \\
\hline
\end{tabular}

A análise descritiva das respostas dos enfermeiros frente a "Escala de Autoavaliação sobre Profissionalismo e Comunicação Interpessoal entre Enfermeiros e Pacientes" mostrou que estes, na maioria das vezes, responderam "sempre" nos itens 2, 3, 4, 5 e 10 (Figura 1). A maioria dos enfermeiros (64,9\%) classificou seu nível de profissionalismo como de moderado a alto ( $\mathrm{Ta}$ bela 3).

Tabela 3. Análise descritiva das respostas dos participantes em cada item da "Escala de Autoavaliação sobre Profissionalismo e Comunicação Interpessoal entre Enfermeiros e Pacientes". Jundiaí, SP, 2016.

\begin{tabular}{|c|c|c|c|c|c|c|c|c|c|c|}
\hline & \multicolumn{2}{|c|}{ Nunca } & \multicolumn{2}{|c|}{ Raramente } & \multicolumn{2}{|c|}{$\begin{array}{l}\text { Algumas } \\
\text { vezes }\end{array}$} & \multicolumn{2}{|c|}{$\begin{array}{l}\text { Frequente- } \\
\text { mente }\end{array}$} & \multicolumn{2}{|c|}{ Sempre } \\
\hline & $\mathrm{n}$ & $\%$ & $\mathrm{n}$ & $\%$ & $n$ & $\%$ & $n$ & $\%$ & $n$ & $\%$ \\
\hline 1 & 2 & 1,2 & 8 & 4,7 & 36 & 21,1 & 73 & 42,7 & 52 & 30,4 \\
\hline 2 & 1 & 0,6 & 2 & 1,2 & 7 & 4,1 & 46 & 26,9 & 115 & 67,3 \\
\hline 3 & 6 & 3,5 & - & - & 2 & 1,2 & 42 & 24,6 & 121 & 70,8 \\
\hline 4 & 2 & 1,2 & 3 & 1,8 & 15 & 8,8 & 73 & 42,7 & 78 & 45,6 \\
\hline 5 & - & - & 4 & 2,3 & 5 & 2,9 & 58 & 33,9 & 104 & 60,8 \\
\hline 6 & - & - & 3 & 1,8 & 18 & 10,5 & 57 & 33,3 & 93 & 54,4 \\
\hline 7 & 6 & 3,5 & 16 & 9,4 & 38 & 22,2 & 71 & 41,5 & 40 & 23,4 \\
\hline 8 & 3 & 1,8 & 8 & 4,7 & 22 & 12,9 & 74 & 43,3 & 64 & 37,4 \\
\hline 9 & - & - & 2 & 1,2 & 24 & 14,0 & 66 & 38,6 & 79 & 46,2 \\
\hline \multirow[t]{2}{*}{10} & - & - & 2 & 1,2 & 2 & 1,2 & 41 & 24,0 & 126 & 73,7 \\
\hline & \multicolumn{2}{|c|}{ Baixo } & \multicolumn{2}{|c|}{$\begin{array}{c}\text { Baixo a } \\
\text { Moderado }\end{array}$} & \multicolumn{2}{|c|}{ Moderado } & \multicolumn{2}{|c|}{$\begin{array}{l}\text { Moderado a } \\
\text { Alto }\end{array}$} & \multicolumn{2}{|c|}{ Alto } \\
\hline 11 & - & - & - & - & 23 & 13,5 & 111 & 64,9 & 37 & 21,6 \\
\hline
\end{tabular}

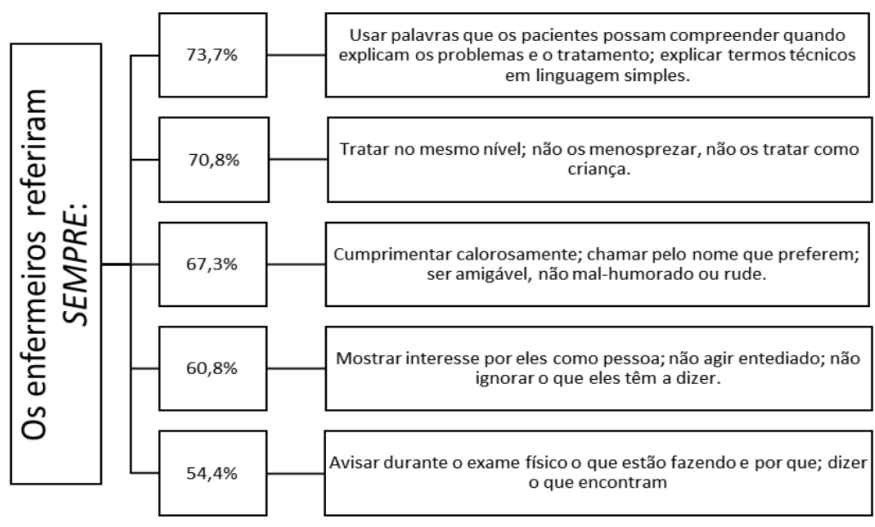

Figura 1. Representação gráfica dos itens respondidos "sempre" pelos enfermeiros. Jundiaí, SP, 2016. 
$\mathrm{Na}$ correlação entre os fatores da "Escala de Autoavaliação sobre Profissionalismo e Comunicação Interpessoal entre Enfermeiros e Pacientes" e as variáveis numéricas tempo de formação, idade e renda em salários mínimos, encontrou-se diferença estatisticamente significativa na comparação do fator Habilidade Interpessoal com a idade $(p=0,05)$. A correlação foi positiva e fraca ( $r$ entre 0,10 a 0,30) e quanto maior a idade, melhor a autoavaliação do enfermeiro em relação a habilidade interpessoal (Tabela 4).

Encontrou-se uma tendência de significância na correlação do tempo de formação com o profissionalismo $(p=0,10)$. A correlação foi positiva e fraca e quanto maior o tempo de formação, melhor a autoavaliação do profissional em relação ao seu profissionalismo.

Tabela 4. Correlação entre os fatores da "Escala de Autoavaliação sobre Profissionalismo e Comunicação Interpessoal entre Enfermeiros e Pacientes" e as variáveis numéricas tempo de formação, idade e renda em salários mínimos. Jundiaí, SP, 2016.

\begin{tabular}{|c|c|c|c|c|c|c|c|c|}
\hline & \multicolumn{2}{|c|}{$\begin{array}{l}\text { Habilidade } \\
\text { Interpessoal }\end{array}$} & \multicolumn{2}{|c|}{$\begin{array}{l}\text { Troca de } \\
\text { informações }\end{array}$} & \multicolumn{2}{|c|}{$\begin{array}{l}\text { Sinceridade } \\
\text { na relação }\end{array}$} & \multicolumn{2}{|c|}{$\begin{array}{l}\text { Profissiona- } \\
\text { lismo }\end{array}$} \\
\hline & $r$ & p-valor & $r$ & p-valor & $r$ & p-valor & $r$ & $p$-valor \\
\hline $\begin{array}{l}\text { Tempo } \\
\text { de } \\
\text { forma- } \\
\text { Ção }\end{array}$ & 0,03 & 0.70 & 0,04 & 0,63 & 0,11 & 0,14 & 0,13 & 0,10 \\
\hline Idade & 0,15 & 0,05 & 0,04 & 0,54 & 0,04 & 0,65 & 0,05 & 0.49 \\
\hline Renda & $-0,07$ & 0,35 & $-0,10$ & 0,22 & $-0,05$ & 0,55 & 0,01 & 0,87 \\
\hline
\end{tabular}

Na associação das variáveis sociodemográficas com os fatores da "Escala de Autoavaliação sobre Profissionalismo e Comunicação Interpessoal entre Enfermeiros e Pacientes", houve diferença estatisticamente significativa na comparação entre o fator sinceridade nas relações e a cor autorreferida $(p=0,04)$, mostrando que os participantes que se declararam como pardos ou negros se percebem mais sinceros na relação com os pacientes (Tabela 5).

Encontrou-se tendências de significância nas associações do fator habilidade interpessoal com a escolaridade $(p=0,12)$, do fator troca de informação com a religião $(p=0,12)$, do fator sinceridade na relação com sexo $(p=0,08)$, unidade de atuação $(p=0,11)$ e religião $(p=0,11)$, do fator profissionalismo com a escolaridade $(p=0,12)$. Esses dados mostram que enfermeiros com mestrado se sentem mais habilidosos na relação interpessoal com o paciente e mais profissionais, enfermeiros sem religião ou sem religiões tradicionais sentem-se que transmitem e recebem melhor as informações durante o exame físico, consideram-se mais sinceros na relação os profissionais do sexo feminino, que atuam em unidade básica de saúde e católicos.

Tabela 5. Associação das características da amostra com os fatores da "Escala de Autoavaliação sobre Profissionalismo e Comunicação Interpessoal entre Enfermeiros e Pacientes". Jundiaí, SP, 2016.

\begin{tabular}{|c|c|c|c|c|c|c|c|c|c|c|c|c|}
\hline \multirow{2}{*}{$\begin{array}{l}\text { Kruskal- } \\
\text { - Wallis/ } \\
\text {-Mann- } \\
\text { - Whit- } \\
\text { ney }\end{array}$} & \multicolumn{3}{|c|}{$\begin{array}{l}\text { Habilidade Inter- } \\
\text { pessoal }\end{array}$} & \multicolumn{3}{|c|}{$\begin{array}{l}\text { Troca de Infor- } \\
\text { mação }\end{array}$} & \multicolumn{3}{|c|}{$\begin{array}{l}\text { Sinceridade na } \\
\text { relação }\end{array}$} & \multicolumn{3}{|c|}{ Profissionalismo } \\
\hline & $\begin{array}{l}\text { Mé- } \\
\text { dia }\end{array}$ & Dp & $\begin{array}{l}\text { p- } \\
\text { va- } \\
\text { lor }\end{array}$ & $\begin{array}{l}\text { Mé- } \\
\text { dia }\end{array}$ & Dp & $\begin{array}{l}\text { p- } \\
\text { va- } \\
\text { lor }\end{array}$ & $\begin{array}{l}\text { Mé- } \\
\text { dia }\end{array}$ & Dp & $\begin{array}{c}p^{-} \\
\text {valor }\end{array}$ & $\begin{array}{l}\text { Mé- } \\
\text { dia }\end{array}$ & Dp & $\begin{array}{l}\text { p- } \\
\text { va- } \\
\text { lor }\end{array}$ \\
\hline Sexo* & & & 0,23 & & & 0,14 & & & 0,08 & & & 0,53 \\
\hline
\end{tabular}

$\begin{array}{lllllllll}\begin{array}{l}\text { Femi- } \\ \text { nino }\end{array} & 18,08 & 2,23 & 12,30 & 2,19 & 8,32 & 1,28 & 8,79 & 0,84\end{array}$
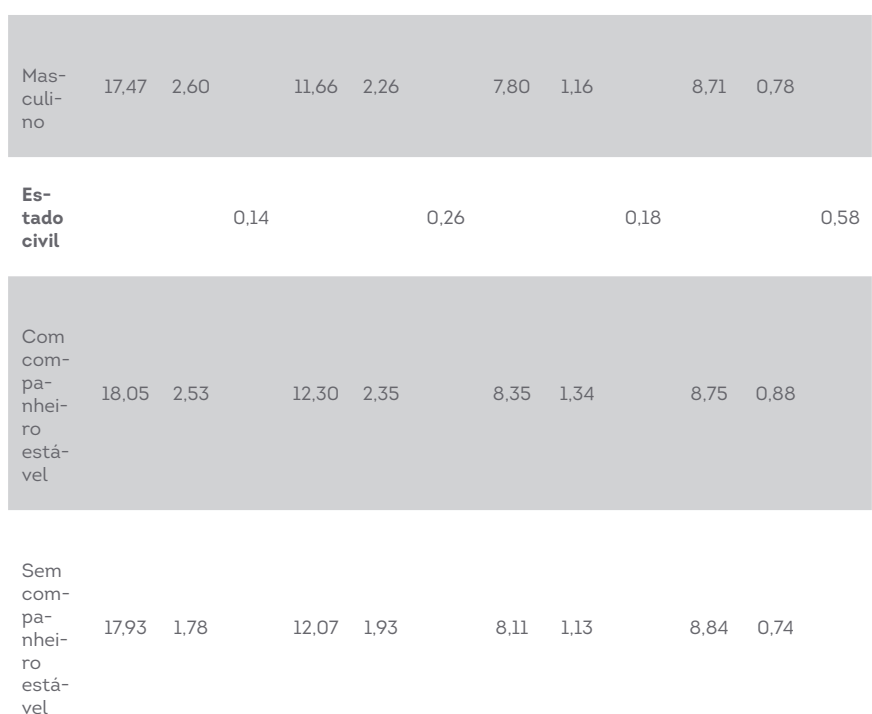

$\begin{array}{lllll}\begin{array}{l}\text { Esco- } \\ \text { lari- } \\ \text { dade }\end{array} & 0,12 & 0,55 & 0,27 & 0,12 \\ \end{array}$
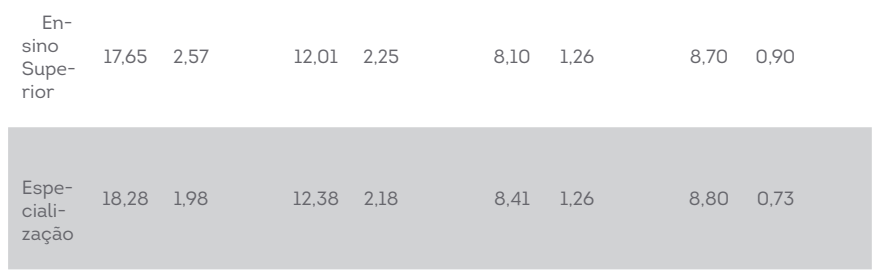

$\begin{array}{lllllllll}\begin{array}{l}\text { Mes- } \\ \text { trado }\end{array} & 18,70 & 1,63 & 12,60 & 1,89 & 8,30 & 1,41 & 9,30 & 0,94\end{array}$

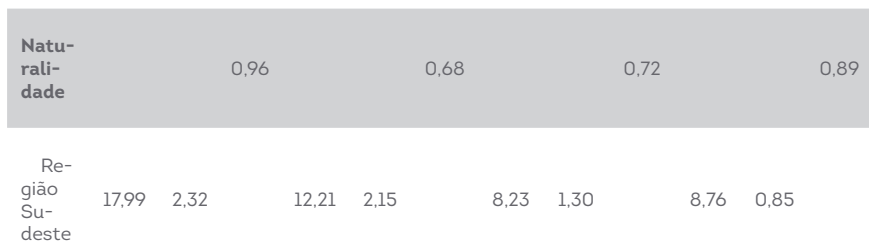



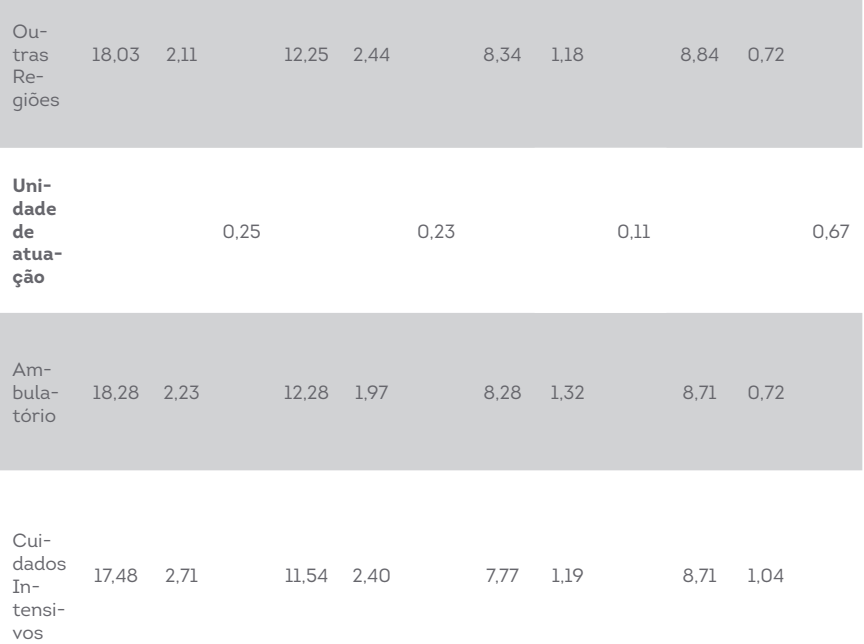

\begin{tabular}{|c|c|c|c|c|c|c|c|c|}
\hline $\begin{array}{l}\text { En- } \\
\text { fer- } \\
\text { maria }\end{array}$ & 18,31 & 2,16 & 12,47 & 1,92 & 8,40 & 1,28 & 8,89 & 0,76 \\
\hline
\end{tabular}

\begin{tabular}{l}
$\begin{array}{l}\text { Pron- } \\
\text { to } \\
\text { So- } \\
\text { corro }\end{array}$ \\
\hline
\end{tabular}
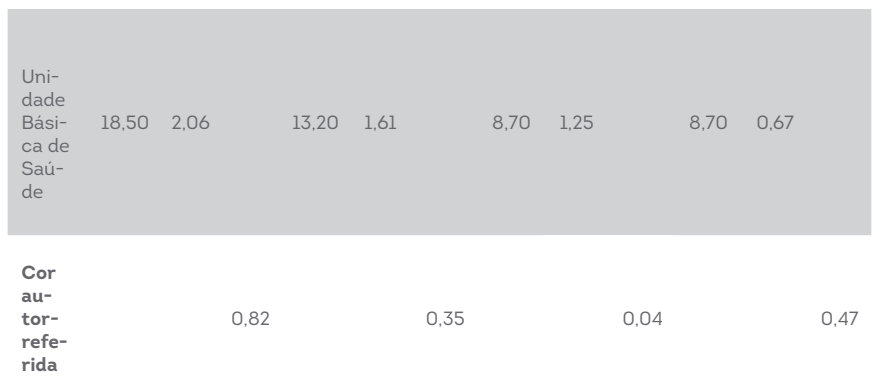

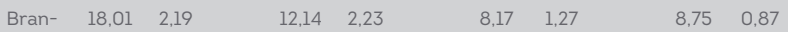

ca

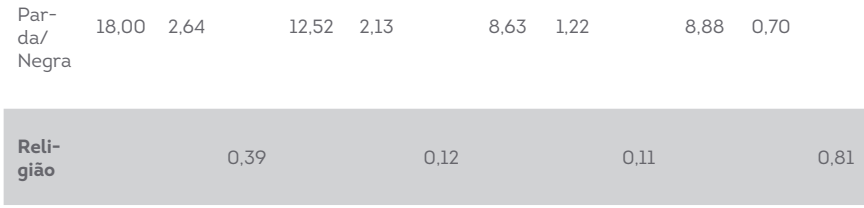

$\begin{array}{lllllllll}\text { Cató- } & 18,04 & 2,37 & 12,40 & 2,42 & 8,45 & 1,20 & 8,77 & 0,85\end{array}$

lico

$\begin{array}{cccccccccc}\begin{array}{c}\text { Es- } \\ \text { pirita }\end{array} & 17,63 & 1,89 & 11,52 & 2,16 & 7,68 & 1,49 & 8,68 & 1,05\end{array}$

$\begin{array}{lllllllll}\begin{array}{l}\text { Evan- } \\ \text { géli- } \\ \text { co }\end{array} & 17,89 & 2,48 & 11,94 & 1,85 & 8,10 & 1,26 & 8,78 & 0,66 \\ \end{array}$

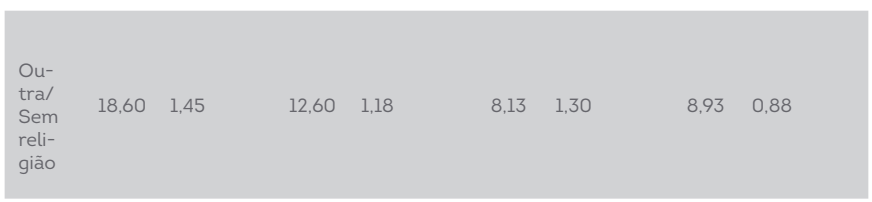

\section{DISCUSSÃO}

Encontrou-se neste estudo que os enfermeiros se perceberam com moderado a alto profissionalismo e competentes na comunicação profissional-paciente; para discutir esse dado é necessário discursar sobre autoavaliação, percepção e fundamentos da comunicação. A autoavaliação da comunicação interpessoal do enfermeiro é um processo bem complexo, importante e uma etapa necessária de sensibilização e percepção para uma posterior intervenção ou aperfeiçoamento do profissional; entretanto possui alguns desafios e limitações.

Quando os indivíduos usam gestos para ilustrar a fala, posam para fotografias ou selecionam a vestimenta do dia, geralmente há um alto nível de consciência e controle nessas ações. Entretanto, maneirismos nervosos, dilatação da pupila e comportamentos imitativos de um parceiro de interação são exemplos de comportamentos que muitas vezes acontecem fora da consciência e do controle das pessoas. Além das expressões, a decodificação dos comportamentos não-verbais dos outros também é realizada em vários graus de consciência ${ }^{(7)}$. Essas são características importantes do processo de comunicação que dificultam a autoavaliação, pois é necessário o desenvolvimento da habilidade de perceber-se e perceber o outro.

A escala utilizada neste estudo aborda alguns comportamentos que podem acontecer de maneira automática no dia-a-dia do profissional e na esfera inconsciente. Um exemplo de um comportamento que frequentemente é realizado no automático e, portanto, precisa de treino para identificação correta, é o resultado que $73,7 \%$ dos enfermeiros afirmaram "sempre" utilizar palavras adequadas e compreensiveis com os pacientes. Essa habilidade comunicacional não é tão simples quanto parece, para uma avaliação mais real dessa adequação, o enfermeiro deve estar atento ao feedback não verbal do paciente, principalmente para expressões de dúvida e satisfação, além de validar as informações dadas solicitando que o paciente verbalize o que foi informado/aprendido.

Percepção exige treino, conhecimento dos fundamentos da comunicação humana, autoconhecimento e autoaceitação das dificuldades e das fortalezas para uma visão das relações interpessoais mais próxima do 
real ${ }^{(2)}$. Frente a isso, é imprescindivel que a autoavaliação seja uma prática constante nas instituições de saúde, favorecendo a reflexão do profissional sobre seus avanços e dificuldades acerca de seu profissionalismo ${ }^{(8)}$. Trabalhar a autoavaliação do profissional é fundamental. É importante que o profissional constantemente analise criticamente o próprio trabalho, compare os resultados alcançados com os esperados e pense em novas estratégias ${ }^{(9)}$.

Um estudo realizado com membros da equipe de enfermagem por meio da observação e problematização, constatou que houve uma deficiência na autocrítica dos participantes e que houve uma tendência em apontar os outros como não colaborativos, e não a si próprio, e propor soluções e mudanças de comportamento para os outros e nunca para si(10).

Aprender a aprender é um processo longo e essencial para profissionais que se propõem a cuidar do outro. Pesquisadores ${ }^{(11)}$ relatam que os profissionais que lidam com momentos difíceis de risco de morte do paciente devem desenvolver a sensibilidade de reconhecer a necessidade de cada paciente e que isso requer respeito pela condição humana e preparo contínuo. Ao adotarem um modo de agir mais empático, os profissionais da saúde ampliam a receptividade e a disponibilidade para escutar, tocar e dialogar.

Os enfermeiros durante sua formação devem adquirir conhecimentos necessários para sua atuação profissional com foco na garantia de uma atenção integral e humanizada aos seus pacientes promovendo qualidade e segurança na assistência. Maneiras interativas de ensino, diferentes das convencionais, inclusive com simulação da prática, vídeos e jogos educativos, podem resultar em uma aprendizagem mais significativa de conceitos essenciais para o enfermeiro(12).

Além do que foi discutido, neste estudo foi encontrado que quanto maior a idade, o tempo de formação e a escolaridade do enfermeiro melhor foi a autoavaliação em relação a competência em comunicação interpessoal. Esse dado reflete a importância tanto da formação, da educação continuada e da atualização quanto do aprendizado com as experiências vivenciadas ao longo dos anos para uma melhor assistência do enfermeiro. Aprender com as oportunidades e com as experiências também se relaciona com a etapa inicial de perceber o outro e perceber-se, o que reforça a importância deste estudo: estimular a autoavaliação do enfermeiro.

\section{Limitações do estudo}

As limitações deste estudo consistem na seleção não aleatória dos participantes e na natureza transversal do estudo, por meio do qual são possíveis apenas associações. Tendências de significância produzem inferências fracas, mas são necessárias em estudos novos, com instrumentos inéditos, com amostras pequenas e quando tem-se a intenção de direcionar posicionamentos e realizar outros estudos sobre o tema.

\section{Contribuição do estudo para a prática}

Os resultados encontrados favorecem a avaliação e a reflexão sobre a complexidade e importância do desenvolvimento da competência em comunicação interpessoal do enfermeiro durante o exame físico. Reflexões geram ações e melhoram a qualidade da assistência do pensar, fazer e agir do enfermeiro. É importante também ressaltar a contribuição deste estudo na valorização da escolaridade e da experiência do enfermeiro para melhorar sua competência em comunicação.

\section{CONCLUSÃO}

Os enfermeiros, em geral, perceberam-se com moderado a alto profissionalismo e competentes na comunicação interpessoal com o paciente durante o exame físico. Relataram que durante o exame físico com o paciente utilizam palavras adequadas e compreensíveis usando uma linguagem simples, trata-os no mesmo nível, chama-os pelo nome que preferem, são amigáveis, bem-humorados, mostram interesse por eles, não ignoram o que falam, não agem entediados, e avisam o que estão fazendo e o porquê, e o que encontram.

Os fatores que interferiram na competência em comunicação interpessoal e profissionalismo do enfermeiro foram: idade, cor autorreferida, tempo de formação, escolaridade, religião, sexo e unidade de atuação; sendo assim, ao conhecer quais fatores se relacionam com a comunicação interpessoal, os profissionais poderão trabalhar seu autoconhecimento, reconhecer suas limitações, buscar alternativas para melhorá-las 
e finalmente, transpor essas melhorarias para sua prática assistencial.

\section{Contribuição dos autores}

Concepção e desenho, análise e interpretação dos dados, redação do artigo, revisão crítica, revisão final: AELP, ACP. Análise e interpretação dos dados, redação do artigo e revisão crítica: FCA, TJP.

Conflito de Interesse: não há.

\section{REFERÊNCIAS}

1. Silva MJP. Comunicação tem remédio: a comunicação nas relações Interpessoais em saúde. 10ạ ed. São Paulo: Loyola; 2015

2. Moscovici F. Desenvolvimento interpessoal: treinamento em grupo. 23ạ ed. Rio de Janeiro: José Olympio; 2015

Stefanelli MC, Carvalho EC. A comunicação nos diferentes contextos da enfermagem. $2^{\text {a }}$ ed. Barueri: Manole; 2012

3. Puggina AC, Silva MJ. Ética no cuidado e nas relações: premissas para um cuidar mais humano. Rev Min Enferm [Internet]. 2009 [cited 2019 Abr 4];13(4):599-605. Available from: http://www.reme org.br/artigo/detalhes/229

4. Santos N, Veiga P. Andrade R. Importância da anamnese e do exame físico para o cuidado do enfermeiro. Rev Bras Enferm [Internet]. 2011 [cited 2019 Abr 4];64(2):355-8. Available from: http://dx.doi. org/10.1590/S0034-71672011000200021

5. Pereira TJ, Puggina AC. Validation of the self-assessment of communication skills and professionalism for nurses. Rev Bras Enferm [Internet]. 2017 [cited 2019 Abr 4];70(3):58894. Available from: http://dx.doi.org/10.1590/00347167-2016-0133

6. Knapp ML, Hall JA. Nonverbal communication in human interaction. 7ạ ed. Belmont: Wadsworth
Publishing; 2009.

7. Ribeiro EAG, Souza NA. A auto-avaliação no curso de pedagogia: do real ao desejável. Londrina: Universidade Estadual de Londrina; 2007

8. Ribeiro EAG, Punhagui GC, Souza NA. Autoavaliação x Autonotação - aproximação e afastamentos na formação de professores autorregulados. Educação. $2014 ; 39(2): 403-14$

9. Bergamin MD, Prado C. Problematização do trabalho em equipe em enfermagem: relato de experiência. Rev Bras Enf [Internet]. 2013 [cited 2019 Abr 4];66(1):1347. Available from: https://dx.doi.org/10.1590/S0034-

\section{1}

10. Silveira RS, Lunardi VL, Lunardi Filho WD, Oliveira AMN. Uma tentativa de humanizar a relação da equipe de enfermagem com a familia de pacientes internados na UTI. Texto Contexto Enferm [Internet]. 2005 [cited 2019 Abr 4]:14(esp):125-30. Available from: http:// dx.doi.org/10.1590/S0104-07072005000500016

11. Lira ALBC, Fernandes MICD, Costa IA, Silva RSC. Estratégia de aprimoramento do ensino do exame fisico. Enfem. Foco [Internet]. 2015 [cited 2019 Jul 04];6(1/4):57-61. Available from: http://revista.cofen gov.br/index.php/enfermagem/article/view/578/260

RECEBIDO: 04/04/2019

ACEITO: $25 / 7 / 2019$ 